\section{FOX: MODULAR APPROACH TO STRUCTURE SOLUTION USING POWDER DATA}

R. Cerny $^{1}$ V. Favre-Nicolin ${ }^{2}$

${ }^{1}$ University of Geneva Laboratory of Crystallography 24, Quai ErnestAnsermet GENEVA 4 CH-1211 SWITZERLAND ${ }^{2}$ ESRF, BP 220, F-38043 Grenoble Cedex, France

FOX [1] is a program for $a b$ initio structure solution from powder data using global optimization algorithms working in direct space. It is a modular program, capable of using several criteria for evaluating each trial configuration. The program is optimized for inorganic materials, with the possibility of describing building blocks in the sample, and with an automatic, adaptive handling of special positions and sharing of identical atoms between neighboring building blocks (dynamical occupancy correction). It has also been tested for organic materials with a few specific optimizations to increase model convergence using improved moves. The building blocks (polyhedrons or molecules) are described with their internal coordinates (Z-matrices), thus allowing natural constraints on interatomic distances and angles. Several algorithms (Simulated Annealing, Parallel Tempering) are currently available. The program is based on an object-oriented crystallographic library ObjCryst++ (programming language $\mathrm{c}++$ ); developed by us. The program and the library are available for Linux and Windows platforms on http://objcryst.sourceforge.net. Structures with the complexity up to 22 independent atoms are routinely solved. Further global optimization algorithms and cost functions will be added soon, and combined search in direct and reciprocal spaces is our further direction of development.

References

[1] Favre-Nicolin V. And Cerny R., FOX \& ObjCryst++ : new object-oriented tools for crystal structure determination. J. Appl. Cryst. - computer programs, in preparation. see also : http://objcryst.sourceforge.net/ and Book of Abstract of the ECM 20, Krakow 2001, p. 135.

\section{Keywords: POWDER DIFFRACTION, STRUCTURE SOLUTION,} GLOBAL OPTIMIZATION

\section{Acta Cryst. (2002). A58 (Supplement), C266}

\section{EXPO2002: THE NEW HEIR OF EXPO}

A.G.G. Moliterni ${ }^{1}$ A. Altomare ${ }^{1}$ R. Caliandro ${ }^{1}$ C. Cuocci $^{2}$ C. Giacovazzo ${ }^{1,2}$ A. ${\text { Guagliardi }{ }^{1} \text { R. Rizzi }}^{1}$

Institute of Crystallography - CNR C/o Dipartimento Geomineralogico Campus Universitario Via Orabona, 4 BARI ITALY 70125 ITALY

${ }^{1}$ Institute of Crystallography, CNR, c/o Dipartimento Geomineralogico, Campus Universitario, 70125 Bari, Italy ${ }^{2}$ Dipartimento Geomineralogico,

Universita di Bari, Campus Universitario, 70125 Bari, Italy

EXPO2002, the evolution of EXPO [Altomare et al. (1999). J. Appl. Cryst. 32, 339-340], is a package aimed at solving ab-initio and at refining crystal structures from a unindexed powder diffraction pattern. The new implemented procedures have greatly enhanced the originary power of EXPO. Among them we quote: 1) new techniques for indexing, via the package N-TREOR [Altomare et al., (2000). J. Appl. Cryst. 33, 1180-1186]; 2) a random partition of the integrated intensities in the full pattern decomposition process [Altomare et al., (2001). J. Appl. Cryst. 34, 704-709], able to overcome the tendency of the Le Bail algorithm [Le Bail et al., (1988). Mater. Res. Bull. 23, 447-452] to equipartition the intensities of strongly overlapping reflections; 3 ) the realspace techniques, already used for the ab-initio crystal structure solution of proteins and optimised for powder diffraction data [Altomare et al., (2002). J. Appl. Cryst. 35. In Press]; 4) the POLPO procedure [Altomare et al., (2000). J. Appl. Cryst. 33, 1305-1310; Altomare et al., (2002). J. Appl. Cryst., Submitted] exploiting the prior knowledge of the cation coordination and of their connectivity; 5) a new peak labelling procedure using the crystal chemical knowledge as prior information [Altomare et al., (2002). J. Appl. Cryst. 35, 2127.]; 6) a new Rietveld [Rietveld, H.M. (1969). J. Appl. Cryst. 2, 65-71] module for structure refinement. A friendly GUI allows to control the refinement strategy and supply geometrical restraints. The main features and applications of the new approaches on a set of test structures will be presented.
Acta Cryst. (2002). A58 (Supplement), C266

AB-INITIO SOLUTION OF INCOMMENSURATE MODULATED STRUCTURES FROM POWDER DIFFRACTION - A SIMULATION J. R. Chen ${ }^{1}$ Y. X. Gu ${ }^{2}$ H. F. Fan ${ }^{3}$

Institute of Physics Chinese Academy of Sciences, P. R.China \#8 Southern 3rd St. ZhongGuanCun BEIJING 100080 CHINA

Powder diffraction data of the high-Tc superconductor Bi-2212 were simulated based on the experimental single-crystal X-ray diffraction data by merging together unique reflections with diffraction angles (theta) closer to each other than $0.02^{\circ}$. The data set contains 1879 reflections, most of them are overlapped. There are three types of overlapping, i.e. (i) overlapping of main and main reflections; (ii) overlapping of satellite and satellite reflections and (iii) overlapping of main and satellite reflections. Many overlapped reflections are mixers of all three types of overlapping. There are 89 of the total 543 main reflections without overlapping with satellites and 295 of the total 1336 satellites without overlapping with main reflections. The third type of overlapping was first separated according to the ratio between the average intensity of the 89 main reflections and that of the 295 satellites. Then the first two kinds of overlapping were treated by uniformly partitioning. Heavy-atom sites in the basic/average structure were found using the decomposed main reflections by the direct-method program SAPI. With phases calculated from the heavy atoms and structure-factor magnitudes of the decomposed main and satellite reflections, phases of the satellites were derived by the multidimensional direct-method program DIMS. Then the program VEC was used to calculate 2-dimensional sections of the 4D-Fourier map, which revealed modulation of all metal atoms as well as the saw-tooth modulation of the oxygen atom on bismuth layer. No assumed model of either the basic structure or the modulation is needed in the process.

\section{Keywords: POWDER DIFFRACTION, DIRECT METHODS, INCOMMENSURATE STRUCTURES}

\section{Acta Cryst. (2002). A58 (Supplement), C266 \\ AB-INITIO STRUCTURE DETERMINATION OF $\mathrm{LiBi}_{3} \mathrm{~V}_{2} \mathrm{O}_{10} \mathrm{BY}$ POWDER X-RAY DIFFRACTION \\ D. Porob T. N. Guru Row \\ Indian Institute of Science Solid State and Structural Chemistry Unit C V Raman Avenue BANGALORE KARNATAKA 560012 INDIA}

The metal doped bismuth vanadium oxides, $\mathrm{Bi}_{2} \mathrm{M}_{\mathrm{x}} \mathrm{V}_{1-\mathrm{x}} \mathrm{O}_{\mathrm{y}}$, BIMEVOX exhibit attractive oxide ion conductivity. A new compound $\mathrm{LiBi}_{3} \mathrm{~V}_{2} \mathrm{O}_{10}$, in the solid solution of $\mathrm{Li}_{2} \mathrm{O}-\mathrm{Bi}_{2} \mathrm{O}_{3}-\mathrm{V}_{2} \mathrm{O}_{5}$ ternary system has been synthesized by solid state reaction. Since the phase decomposes on melting, growth of single crystal is not feasible.

The structure of $\mathrm{LiBi}_{3} \mathrm{~V}_{2} \mathrm{O}_{10}$ was determined by ab-initio methods using highresolution powder X-ray diffraction data on a laboratory source. The compound is triclinic, space group $P-1, \mathrm{a}=9.2074(2) \AA, \mathrm{b}=5.5371(1) \AA$, $\mathrm{c}=$ $12.6005(2) \AA, \alpha=68.701(1)^{\circ}, \beta=125.828(1)^{\circ}, \gamma=118.230(1)^{\circ}$ and $\mathrm{V}=$ 455.53(1) $\AA^{3}$.

Rietveld refinements result in $\mathrm{R}(\mathrm{p})=7.94 \%, \mathrm{R}(\mathrm{wp})=10.84 \%, \mathrm{R}(\mathrm{I}, \mathrm{hkl})=$ $5.50 \%$ for 71 structural parameters and 4799 data points. The structure is similar to that of $\gamma-\mathrm{NaBi}_{3} \mathrm{~V}_{2} \mathrm{O}_{10}$ polymorph, built from $\left(\mathrm{Bi}_{2} \mathrm{O}_{2}\right)$ double chains that extend along the $\mathrm{b}$ axis with $\mathrm{VO} 4$ units joining the chains along both a and $\mathrm{b}$ directions. The light atom positions, $\mathrm{Li}$ and $\mathrm{O}$, have been verified by powder neutron diffraction and the lithium coordination substantiated by solid state NMR study.

Keywords: AB-INITIO STRUCTURE, DOPED BISMUTH-VANADATE, X-RAY POWDER 